\title{
Compensation of phase nonlinearity of liquid crystal spatial light modulator for high-resolution wavefront correction
}

H. Zhang

zhxlj2004@163.com

H. Zhou

J. Li

Y. J. Qiao

J. Si

W. Gao
Intelligent Machine Institute, Harbin University of Science and Technology

Intelligent Machine Institute, Harbin University of Science and Technology

Intelligent Machine Institute, Harbin University of Science and Technology

Intelligent Machine Institute, Harbin University of Science and Technology

Intelligent Machine Institute, Harbin University of Science and Technology

Institute of Photonics and Optical Fiber Technology, Harbin University of Science and Technology, No. 52 Xuefu Road, Harbin, Heilongjiang, 150080, China

The ability of phase modulation enables liquid crystal spatial light modulator (LCSLM) to control wavefront. However, the disadvantage of its inherent nonlinear phase response will decrease the wavefront control accuracy. In this paper, a compensation for the nonlinear phase response is proposed based on Inverse Interpolation method. Characteristic curve of phase retardation versus gray levels for a $256 \times 256$ pixels phase-only LCSLM has been measured and calibrated by Inverse Interpolation. A mapping relationship between input gray levels and driving gray levels has been built and recorded by a linear look-up table ANTI2.LUT. The nonlinear error of the phase drops from $15.9 \%$ to $2.42 \%$ by using ANT12.LUT. Further more, the mapping curve of ANTI2.LUT is almost consistent with 290.LUT from the manufacturer, which proved the efficiency of the compensation of phase nonlinearity. Finally, the distorted wavefront caused by a liquid crystal flake is corrected using LCSLM based on ANTI2.LUT. Experimental results show that the peak-valley value of the distorted wavefront decreases from 1.56 $\lambda$ to $0.26 \lambda(\lambda=0.6328 \mu \mathrm{m})$, the root-mean-square value decreases from $0.25 \lambda$ to $0.02 \lambda$ and the Strehl ratio of diffractive spots increases from 0.08 to 0.97 . So LCSLM can be applied to realize high-precision and high-resolution wavefront correction with linear phase response.

[DOI: http://dx.doi.org/10.2971/jeos.2015.15036]

Keywords: Wavefront correction, phase nonlinearity compensation, inverse interpolation, liquid crystal spatial light modulator

\section{INTRODUCTION}

Liquid crystal spatial light modulator (LCSLM) is regarded as a perfect wavefront controller because of its advantages such as low-power consumption, high-resolution, non-mechanical and programming control. As a dynamic diffraction device, LCSLM has wide applications in adaptive optics [1, 2], laser beam shaping and scan [3]-[7], real-time hologram display [8,9] and holographic optical tweezers [10, 11]. However, the inherent nonlinearity of phase versus gray level addressed by LCSLM limits its performance in these applications. Especially in the field of adaptive optics, when LCSLM is used as a wavefront corrector, phase nonlinearity leads to the complex transform of feedback signals, which will reduce the data processing efficiency and further introduce a transform error. To overcome the existed shortage, most manufacturers of LCSLMs have already provided linear lookup tables (LUTs) to meet the requirement for linear driving. However, after many times of operation, the linear look-up tables should be calibrated again to meet the precision requirement. Therefore, it is necessary for users to develop a phase nonlinearity compensation method independently for high-precision wavefront correction.
A method of Gamma correction is a mature method for liquid crystal displays $[12,13]$. In order to satisfy the subjective sense of human eyes to object light, it is necessary to generate a power function relationship between the output light intensity $E(x, y)$ and input signal $D$ in the liquid crystal display, which is called a Gamma curve. It can be expressed as $E(x, y)=D^{\gamma}$. But the actual curve of liquid crystal transmittance versus control voltages show a S-shaped distribution. So the Gamma correction curve can be obtained by a nonlinear transformation of reverse S-shaped point by point. Gamma correction needs a long time-consuming and is not suitable for phase-only LCSLM which asks a linear driving relationship between the phase retardation and the control voltage. In addition, another simple method of phase nonlinear compensation is an approximate correction method for the phase-only LCSLM [14, 15]. An approximate linear length of the curve of the phase retardation versus control voltage can be chosen as the linear work curve. The approximate linear curve can be directly used to drive LCSLM without setting the linear LUT. However, the resolution of control commands cannot meet the requirement of high-resolution phase control. Except for 
above methods, some researchers compensate phase nonlinearity point by point $[16,17]$. The process is to put the measured phase curve and the ideal linear curve into one coordinate system, and then find the mapping relationship of corresponding grayscale values of two groups, at last write the mapping relationship into a LUT to obtain a linear driving to LCSLM. This method increases the resolution of controllable phase, but it is hard to calibrate LCSLM rapidly due to the complex operating process and low efficiency.

This paper proposes a compensation method for the phase nonlinearity based on an Inverse Interpolation method. An inverse interpolation function is used to build the mapping relationship between input gray levels and driving gray levels which is written into a LUT for the linear driving to LCSLM. A $256 \times 256$ pixels phase-only LCSLM from American BNS Company is used as the device of compensation of phase nonlinearity. A high resolution wavefront correction is completed using LCSLM with the linear LUT.

\section{COMPENSATION OF PHASE NONLINEARITY}

According to the birefringence effect, liquid crystal (LC) molecules tilt at angle $\theta$ when an electric field is applied over the LC layer which causes the decrease in effective extraordinary refractive index $n_{e}(\theta)$. The relation between $n_{e}(\theta)$ and $\theta$ can be expressed as

$$
n_{e}(\theta)=\frac{n_{0} n_{e}}{\sqrt{n_{0}^{2} \cos ^{2} \theta+n_{e}^{2} \sin ^{2} \theta}}
$$

where $n_{o}$ and $n_{e}$ are refractive indexes of ordinary and extraordinary light of LC respectively.

According to the theory of refractive index of anisotropic crystals, the liquid crystal material is optically anisotropic which is similar to a uniaxial crystal. A phase retardation $\delta$ will generate by an applied voltage when a beam of light passes through the LC layer, which can be expressed as

$$
\delta=\frac{2 \pi}{\lambda} n_{e f f} L=\frac{2 \pi}{\lambda} \int_{0}^{L} n_{e}(\theta) d z
$$

where $\lambda$ is the wavelength of the incident light, $n_{e f f}$ is the equivalent refractive index and $L$ is the thickness of the LC layer.

The characteristic curve of phase retardation versus driving voltage is shown in Figure 1. Because the nematic LC material only responds to the strength of the electric fields instead of the voltage polarity, both of the control voltages $V_{0} \sim V_{2 \pi}$ and $-V_{0} \sim-V_{2 \pi}$ can cause the same phase retardation and the relationship between phase retardation and voltage is nonlinear. According to the theory of crystal refractive index the phase retardation depends on the change of the equivalent refractive index of the liquid crystal when a beam of light goes through liquid crystal layer. The equivalent refractive index has a nonlinear response on the driving voltage so the relationship of phase retardation versus voltage is nonlinear $[18,19]$. In order

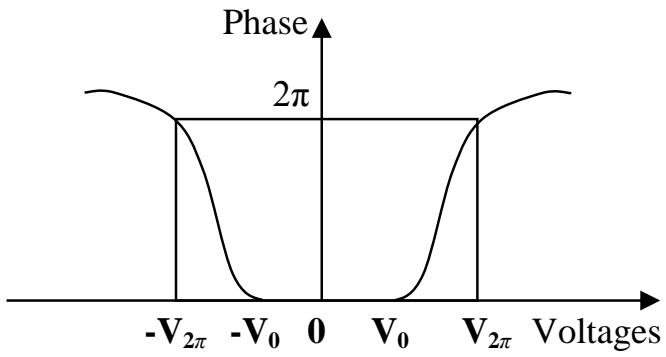

FIC. 1 Curves of phase retardation versus voltage.

to simplify the manipulating of LCSLM, the manufacturers convert the driving voltages of LCSLM into grayscale values which range from 0 to 255 . When loading a gray image with a grayscale value, LCSLM can generate a phase retardation.

The nonlinear function of phase versus gray level is assumed to be a discrete function $y_{i}=f\left(x_{i}\right)(i=1,2,3, \ldots, n)$, where $x_{i}$ is the grayscale value, $y_{i}$ is the phase and the number of discrete data $n$ is less than or equal to 255 . In order to get a smooth interpolation curve, the interpolation polynomial of Cubic Spline which can be constructed by known data $\left[x_{i}, y_{i}\right]$ is shown as follows

$$
\begin{aligned}
P(x)= & f\left(x_{i-1}\right)+f\left[x_{i-1}, x_{i}\right]\left(x-x_{i-1}\right) \\
& +\left[a_{i}\left(x-x_{i-1}\right)+b_{i}\left(x-x_{i}\right)\right]\left(x-x_{i-1}\right)\left(x-x_{i}\right)
\end{aligned}
$$

where $a_{i}$ and $b_{i}$ are interpolation coefficients in the range $\left[x_{i-1}, x_{i}\right]$ and the mean difference of one degree $f\left[x_{i-1}, x_{i}\right]$ is defined as

$$
f\left[x_{i-1}, x_{i}\right]=\frac{f\left(x_{i}\right)-f\left(x_{i-1}\right.}{x_{i}-x_{i-1}}
$$

When some grayscale values $x_{i+1}, x_{i+2}, \ldots, x_{i+k},\left(x_{i+k}<x_{n}\right)$ are interpolated into Eq. (3), the corresponding phase values $y_{i+1}, y_{i+2}, \ldots, y_{i+k}$ can be calculated. If it is needed to inversely solve grayscale values, an inverse interpolation calculation $x_{i}=f^{-1}\left(y_{i}\right)$ can meet the requirement. The inverse interpolation polynomial is as follows

$$
\begin{aligned}
P(x)= & f^{-1}\left(y_{i-1}\right)+f^{-1}\left[y_{i-1}, y_{i}\right]\left(y-y_{i-1}\right) \\
& +\left[c_{i}\left(y-y_{i-1}\right)+d_{i}\left(y-y_{i}\right)\right]\left(y-y_{i-1}\right)\left(y-y_{i}\right)
\end{aligned}
$$

where $c_{i}$ and $d_{i}$ are inverse interpolation coefficients in the range $\left[y_{i-1}, y_{i}\right]$ and $f^{-1}\left[y_{i-1}, y_{i}\right]$ is the mean difference of one degree.

Another ideal linear function relationship between phase and gray level is constructed as below,

$$
Y_{j}=F\left(X_{j}\right)=\frac{2 \pi}{N} X_{j}+Y_{0} \quad(j=1,2,3, \ldots, N)
$$

where $X_{j}$ is the theoretical grayscale, $Y_{j}$ is the phase which will be used as inverse interpolation node, $Y_{0}$ is initial phase and the number of discrete data $N$ is less than or equal to 255 . $Y_{0}$ is dependent on the initial value of measured phases. For instance, $Y_{0}$ equals $\phi$ as the initial value of measured phases is $\phi$.

Phase $Y_{j}$ is substituted into the inverse interpolation polynomial (Eq. (5)), as a result, the inverse interpolation grayscale can be obtained $X_{j}^{\prime}=P\left(Y_{j}\right)$. The mapping relationship 


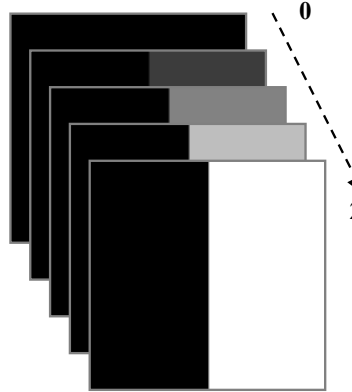

(a)

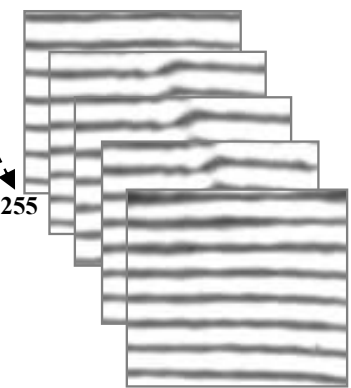

(b)
FIG. 2 Gray images for Twyman-Green interferometry and interferograms (a) gray images; (b) interferograms.

$X_{j}^{\prime}=E\left(X_{j}\right)$ between $X_{j}^{\prime}$ and $X_{j}$ is also established with the aid of the middle variables $Y_{j}$, which will be written into a linear LUT later. $X_{j}$ is regarded as the input gray level from users and $X_{j}^{\prime}$ is the driving gray level which will be converted into voltage. So the linear LUT can be used to compensate phase nonlinear and meet the requirement for linear driving to LCSLM.

\section{EXPERIMENT AND ANALYSIS}

\subsection{Measurement and compensation of phase nonlinearity}

The $256 \times 256$ pixels phase-only reflective LCSLM from American BNS Company is measured by using Twyman-Green interferometry [20]. The gray images are generated as shown in Figure 2(a), in which the grayscale values on the left sides of the gray images are always set to be zero, and the grayscale values of the right sides are increased with an increment of 5 from 0 to 255. 52 gray images are loaded to LCSLM in turn and a group of interference fringe patterns are obtained through the interference measurement as shown in Figure 2(b).

The characteristic curve of phase shift versus gray level can be obtained by calculating through the following expression

$$
\delta_{i}=\frac{2 \pi \Delta_{i}}{\Lambda}
$$

where $\Delta_{i}$ is the moving distance and $\Lambda$ is the width of one period of interference fringes. In Figure 3 the monotonous rising curve of phase response corresponds to the grayscale scope 0 to 127 and the monotonous dropping curve corresponds to 127 to 255 , so there exist two monotonous and symmetrical curves in the range of 0 to 255 .

The phase curve in Figure 3 is nonlinear in the gray level range from 0 to 255 with symmetrical distribution and the maximum phase retardation is $3.16 \pi$. The linear driving relationship expected by users is the linear correspondence between grayscales $0 \sim 255$ and phases $0 \sim 2 \pi$. Due to LCSLM is a reflective type grayscales $0 \sim 128$ can driving phase $0 \sim 2 \pi$. The linear correspondence between grayscales and phase can be calculated through Eq. (6) and the values are as show in column 1 and 2 of Table 1 . But the experimental grayscales from column 3 of Table 1 correspond to phase nonlinearly. The reason of the nonlinear correspondence between experimental grayscales and phases has been explained in Section 2. The

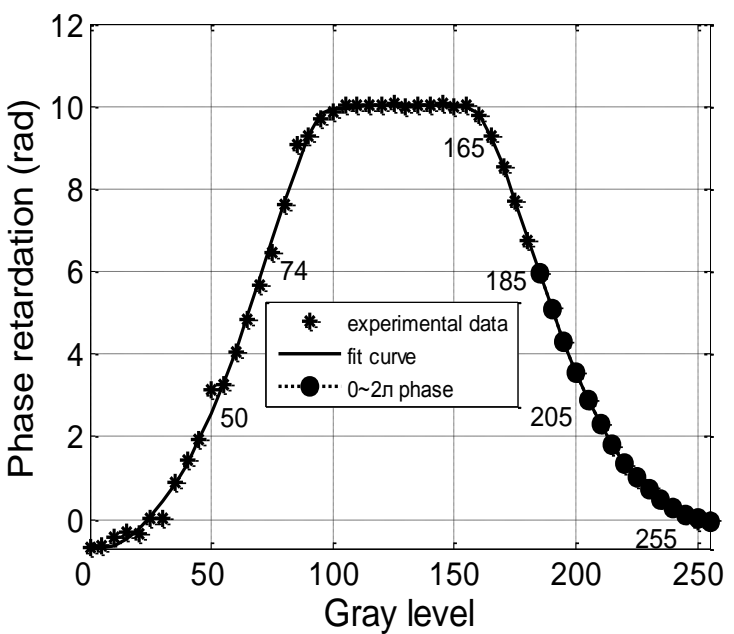

FIG. 3 Characteristic curve of phase modulation versus gray level.

\begin{tabular}{|c|r|r|}
\hline $\begin{array}{c}\text { Phase } \\
\text { (rad) }\end{array}$ & $\begin{array}{r}\text { Theoretical } \\
\text { grayscales }\end{array}$ & $\begin{array}{r}\text { Experimental } \\
\text { grayscales }\end{array}$ \\
\hline 0 & 0 & 26 \\
\hline$\pi / 2$ & 32 & 39 \\
\hline$\pi$ & 64 & 53 \\
\hline $3 \pi / 2$ & 96 & 63 \\
\hline $2 \pi$ & 128 & 71 \\
\hline $2 \pi$ & 128 & 185 \\
\hline $3 \pi / 2$ & 160 & 194 \\
\hline$\pi$ & 192 & 205 \\
\hline$\pi / 2$ & 224 & 221 \\
\hline 0 & 255 & 255 \\
\hline
\end{tabular}

TABLE 1 Theoretical and experimental data of phase versus grayscales.

nonlinear phase response will increase the difficulty of wavefront control. So the phase nonlinearity correction is necessary.

An Inverse Interpolation algorithm can be used to compensate phase nonlinearity. In Figure 3 a part of the phase curve corresponding to gray levels 0 to 70 is chosen as inverse interpolation nodes. In order to reduce the influence from random error, it is necessary to fit the measured data with least squares algorithm and then to subdivide the interpolation nodes to 71 pairs of discrete data $\left[x_{i}, y_{i}\right]$ to decrease the gray level interval from 5 to 1 . The other group of data $\left[X_{i}, Y_{i}\right]$ which has linear correspondence is established by Eq. (6). Phases $Y_{i}$ are used to inversely interpolate the measured phases $y_{i}$ to build the mapping relationship between grayscales $X_{i}$ and $x_{i}$ through Inverse Interpolation. The mapping relationship is written into a look-up table ANTI1.LUT and the curve is as shown in Figure 4(c). The curves of phase retardation before and after using ANTI1.LUT are shown in Figure 4(a) and (b) respectively.

The phase nonlinearity is significantly reduced and the nonlinearity error is reduced from $21.15 \%$ to $2.64 \%$ after compensation. The formula of nonlinearity error is defined as

$$
e=\frac{\left|\delta_{i}-\bar{\delta}_{i}\right|_{\max }}{\delta_{\max }-\delta_{\min }} \times 100 \%
$$

where $\delta_{i}$ is the measured phase, $\bar{\delta}_{i}$ is the ideal value corresponding to $\delta_{i}$ and $\delta_{\max }-\delta_{\min }$ is the maximum of phase retardation. 

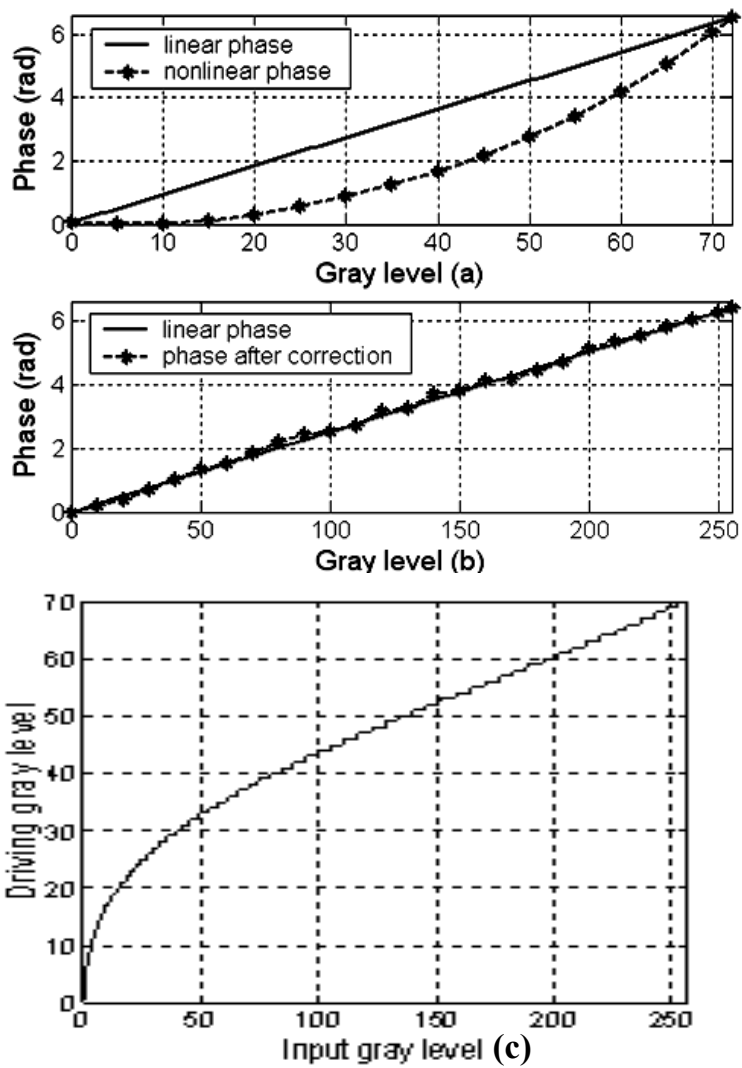

FIG. 4 Phase retardation curves before and after using ANTI1.LUT and mapping curve of ANTI1.LUT (a) Phase retardation curves without ANTI1.LUT; (b) Phase retardation curves with ANTI1.LUT; (c) Mapping curve of ANTI1.LUT.

Therefore, an approximate linear driving to LCSLM can be achieved through the linear look-up table ANTI1.LUT which is built by Inverse Interpolation algorithm. The resolution of controllable phase corresponding to 70 gray levels is increased clearly. The experiment result indicates that phase nonlinearity of LCSLM can be effectively compensated by Inverse Interpolation algorithm.

The linear look-up table 290.LUT of LCSLM from American BNS Company is used as a comparing example to prove the accuracy of Inverse Interpolation algorithm. Firstly, a part of the phase curve in Figure 3 corresponding with grayscales from 185 to 255 is corrected by Inverse Interpolation algorithm and a linear look-up table ANTI2.LUT is built. The mapping relationships of input grayscales and driving grayscales corresponding to 290.LUT and ANTI2.LUT are almost in agreement with each other in Figure 5(c). The phase retardation curves before and after using ANTI2.LUT are shown in Figure $5(\mathrm{a})$ and (b) and the phase nonlinearity error decreases from $15.9 \%$ to $2.42 \%$.

Although the phase values are both $0 \sim 2 \pi$ corresponding to grayscales $0 \sim 70$ and grayscales $185 \sim 255$ respectively, the phase curves in Figure 3 are not completely symmetrical and the initial nonlinearity errors are not similar either. However, both of the nonlinearity errors are decreased to nearly 0.025 using Inverse Interpolation algorithm. The result shows that the nonlinearity correction is effective and a good linear phase response can be realized.
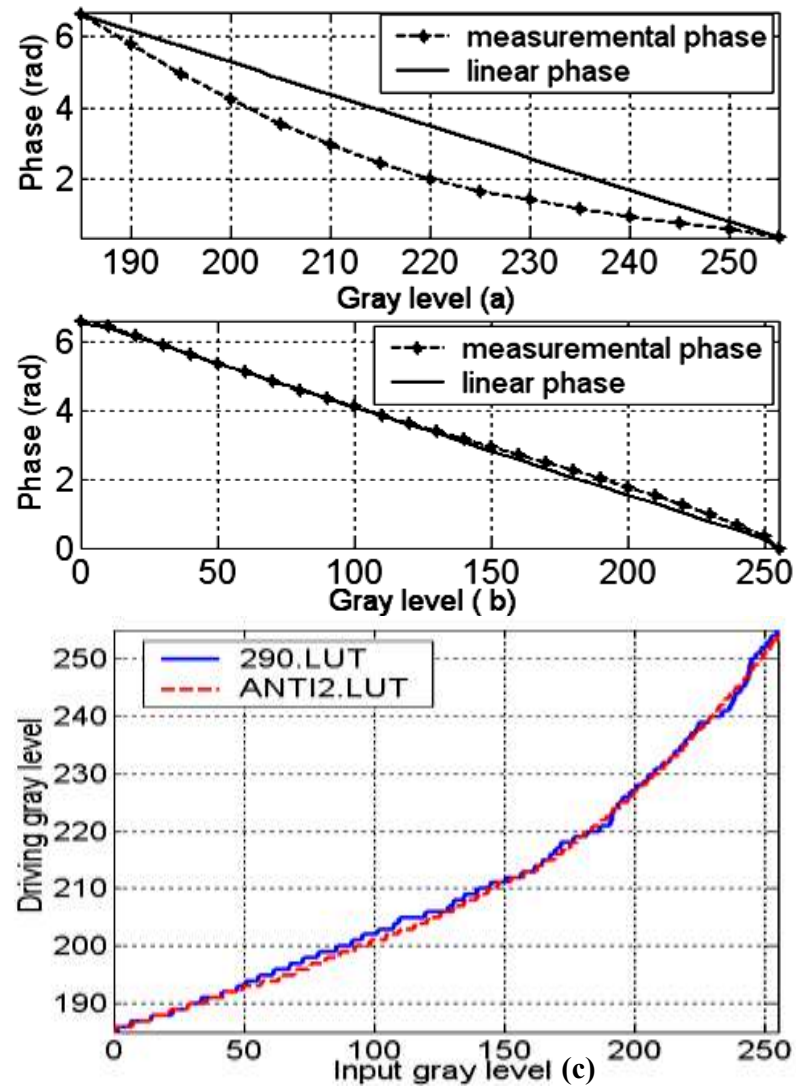

FIG. 5 Phase retardation curves before and after using ANT/2.LUT and mapping curves of 290.LUT and ANTI2.LUT (a) Phase retardation curves without ANTI2.LUT; (b) Phase retardation curves with ANTI2.LUT; (c) Mapping curves corresponding to 290.LUT and ANTI2.LUT.

Experimental results show that Inverse Interpolation algorithm is quite available for LCSLM calibration with simplification and speediness, moreover, it can increase the calibration efficiency of LCSLM.

\subsection{Correction of wavefront distortion}

A reflective LCSLM from American BNS and a Fizeau interferometer AK-100 are used to measure and correct the wavefront aberration as shown in Figure 6. A liquid crystal flake (as shown in Figure 7) is used as a distortion element. A wavefront correction experiment is done by using the linear lookup table ANTI2.LUT in order to prove that LCSLM can be used to realize high-precision and high-resolution wavefront correction under linear driving of phase.

Figure 8(a) shows the fringes from the interference between an part of the edge of the liquid crystal flake and a reference plane wave. The step planes of the edge of the liquid crystal flake causes the fringes produce misalignment and the imperfect surface of the liquid crystal flake also makes the fringes deformed. The PV value of measured wavefront aberration is $1.56 \lambda(\lambda=0.6328 \mu \mathrm{m})$, the RMS value is $0.26 \lambda$ and the Strehl ratio of the diffraction light spots is 0.08 .

According to the principle of phase conjugation a closed-loop feedback control is used to correct the distorted wavefront to a near plane wave. The linear look-up table ANTI2.LUT is used to linearly drive LCSLM. The phase modulation can vary lin- 
Polarizer Liquid crystal flake

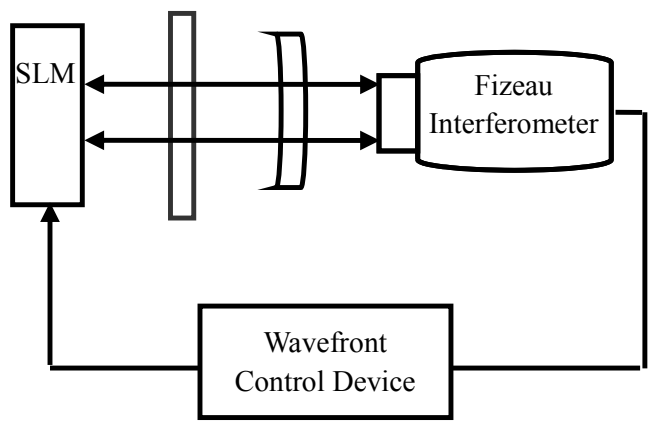

FIG. 6 Experimental setup

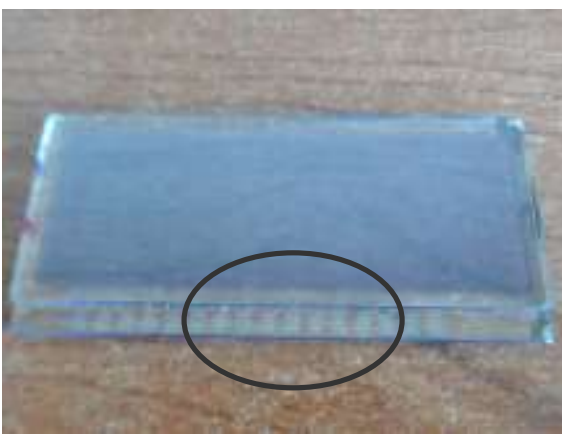

FIG. 7 Liquid crystal flake.

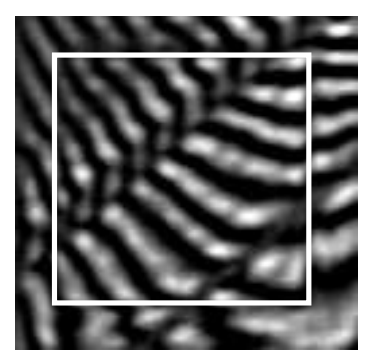

(a)

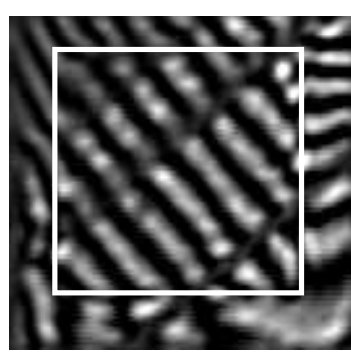

(b)
FIC. 8 Interference fringes before and after correction (a) Fringes before correction; (b) Fringes after correction.

early with the control grayscales through ANTI2.LUT, so the measured wavefront aberration can be directly transformed into a gray level image with phase conjugation information. And then the gray level image is loaded on LCSLM to generate phase modulation. The closed-loop control is performed circularly for five times till reaching the minimum RMS error.

The experiment results are shown in Figure 8 and Figure 9. Figure 8(a) and (b) are interference fringes before and after wavefront correction and Figure 9(a) and (b) are diffraction light spot patterns before and after correction. The PV value of the wavefront aberration decreases from $1.56 \lambda$ to $0.26 \lambda$ after correction, the RMS value decreases from $0.25 \lambda$ to $0.02 \lambda$ and the Strehl ratio increases from 0.08 to 0.97 . The distorted wavefront can be corrected to a near plane wave using LCSLM with the linear look-up table ANTI2.LUT and the closed-loop feedback control.

\section{CONCLUSION}

The phase nonlinearity of a $256 \times 256$ pixels phase-only reflective LCSLM from BNS company is measured and calibrated.

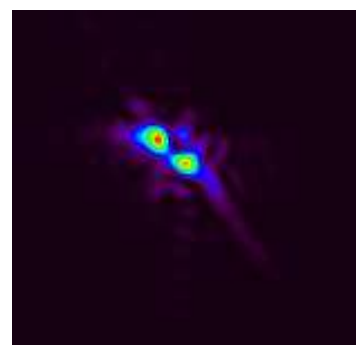

(a)

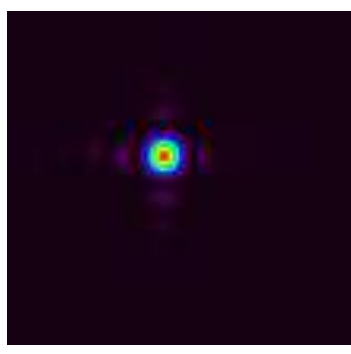

(b)
FIG. 9 Diffraction light spots before and after correction (a) Diffraction light spot before correction; (b) Diffraction light spot after correction.

By using the Inverse Interpolation method to build a linear look-up table ANTI2.LUT, the nonlinear error of phase has been decreased from $15.9 \%$ to $2.42 \%$. When further comparing the linear look-up table ANTI2.LUT obtained in this paper and the table 290.LUT offered by the BNS manufacturer, it shows that the mapping relationships between the input gray levels and driving gray levels in two tables are almost consistent. The results show that we can use Inverse Interpolation method to calibrate the phase nonlinearity of LCSLM once more for linear driving when the look-up table built by the company is not accurate due to the long-time use and is not able to meet the needs update. Finally, by using ANTI2.LUT, the compensation of wavefront distortion from a liquid crystal flake has been realized by a closed-loop feedback control. The experimental results show that the RMS value of the distorted wavefront is reduced by 12.5 times, from $0.25 \lambda$ to $0.02 \lambda$ and the Strehl ratio increases from 0.08 to 0.97 which is close to the diffraction limit. Therefore, the nonlinearity of phase versus driving grayscale of LCSLM can be calibrated independently by users and LCSLM can be used for higher-precision and higher-resolution wavefront correction.

\section{ACKNOWLEDGEMENTS}

This research is supported by National Natural Science Foundations of China of No. 61178069 and No. 61378003.

\section{References}

[1] B. P. Cumming, A. Jesacher, M. J. Booth, T. Wilson, and Min Gu, "Adaptive aberration compensation for three-dimensional microfabrication of photonic crystals in lithium niobate," Opt. Express 19, 9419-9425 (2011).

[2] C. Liu, L. F. Hu, Q. Q. Mu, Z. L.Cao, and L. Xuan, “Open-loop control of liquid-crystal spatial light modulators for vertical atmospheric turbulence wavefront correction," Appl. Opt. 50, 82-89 (2011).

[3] H. Ma, P. Zhou, X. Wang, Y. Ma, F. Xi, X. Xu, and Z. Liu, “Neardiffraction-limited annular flattop beam shaping with dual phase only liquid crystal spatial light modulators," Opt. Express 18, 8251-8260 (2010).

[4] D. Wang, J. Zhang, H. Wang, and Y. Xia, "Variable shape or variable diameter flattop beam tailored by using an adaptive weight FFTbased iterative algorithm and a phase-only liquid crystal spatial light modulator," Opt. Commun. 285, 5044-5050 (2012).

[5] D. Wang, J. Zhang, and H. X. Zhang, "Adaptive-weight iterative algorithm for flat-top laser beam shaping," Opt. Eng. 51, 074301 (2012). 
[6] D. Wang, J. Zhang, Y. Xia, and H. Wang, "Independent and arbitrary generation of spots in the $3 \mathrm{D}$ space domain with computer generated holograms written on a phase-only liquid crystal spatial light modulator," J. Opt. 14, 105704 (2012).

[7] D. Wang, Y.-L. Wu, B.-Q. Jin, P. Jia, and D.-M. Cai, “A phase distribution design method for phased arrays multi-beam independently generating and three-dimensional scanning," IEEE Photonics J. 6, 6802111 (2014).

[8] J. S. Hong, Y. M. Kim, H. J. Choi, J. Hahn, J. H. Park, H. Kim, S.-W. Min, et al., "Three-dimensional display technologies of recent interest: principles, status, and issues," Appl. Opt. 50, H87-H115 (2011).

[9] M. Makowski, I. Ducin, M. Sypek, A. Siemion, A. Siemion, J. Suszek, and A. Kolodziejczyk, "Color image projection based on Fourier holograms," Opt. Lett. 35, 1227-1229 (2010).

[10] M. Persson, D. Engström, A. Frank, J. Backsten, J. Bengtsson, and M. Coksör, "Minimizing intensity fluctuations in dynamic holographic optical tweezers by restricted phase change," Opt. Express 18, 11250-11263 (2010).

[11] J. Andilla, E. Martín-Badosa, and S. Vallmitjana, "Prediction of phase-mostly modulation for holographic optical tweezers," Opt. Commun. 281, 3786-3791 (2008).

[12] K. D. Xiao, C. Y. Fu, K. Dimosthenis, and S. Wuerger, "Visual gamma correction for LCD displays," Displays 32,17-23 (2011).
[13] Y. Y. Wei, H. F. Li, and X. Liu, "Automatic gamma correction system for liquid crystal projector," J. Zhejiang Univ-Sc. A 39, 1727-1729 (2005) in Chinese.

[14] S. R. Harris, "Characterization and application of a liquid crystal beam steering device," Proc. SPIE 4291,109-119 (2001).

[15] L. F. Hu, L. Xuan, Y. J. Liu, Z. L. Cao, D. Y. Li, and Q. Q. Mu, “Phaseonly liquid-crystal spatial light modulator for wavefront correction with high precision," Opt. Express 12, 6403-6409 (2004).

[16] X. J. Zhao, C. L. Liu, D. Y. Zhang, and Y. Q. Luo, “Direct investigation and accurate control of phase profile in liquid-crystal optical-phased array for beam steering," Appl. Optics 52, 71097116 (2013).

[17] S. Reichelt, "Spatially resolved phase-response calibration of liquid-crystal-based spatial light modulators," Appl. Opt. 52, 2610-2618 (2013).

[18] D. Engström, M. Persson, J. Bengtsson, and M. Goksör, "Calibration of spatial light modulators suffering from spatially varying phase response," Opt. Express 21, 16086-16103 (2013).

[19] A. Linnenberger, S. Serati, and J. Stockley, "Advances in optical phased array technology," Proc. SPIE 6304, 63040T (2006).

[20] H. X. Zhang, J. Zhang, L. Y. Wu, "Measurement of phase and intensity by Dual-imaging Interferometry for performance evaluation of liquid crystal spatial light modulator," Optik 122, 1249-1253 (2011). 